\title{
Molecular modeling of zinc paddlewheel molecular complexes and the pores of a flexible metal organic framework
}

\author{
Khalid A. H. Alzahrani ${ }^{1}$. Robert J. Deeth ${ }^{1,2}$
}

Received: 7 January 2016 / Accepted: 26 February 2016 / Published online: 15 March 2016

(C) The Author(s) 2016. This article is published with open access at Springerlink.com

\begin{abstract}
A new all-atom first-principles force field (FF) is constructed for the bimetallic, four-bladed zinc paddlewheel (ZPW) motif. Zinc-ligand interactions are described via Morse functions and the angular geometry at the metal centers is modeled with a pure ligand-ligand repulsion term. The ZPW-FF is principally based on 15 DFT-optimized model systems of general formula $\mathrm{ZnPR} . \mathrm{nL}$, where $\mathrm{ZnP}$ is the base $\mathrm{Zn}_{2}\left(\mathrm{O}_{2} \mathrm{CR}\right)_{4}$ unit, $\mathrm{R}=\mathrm{H}, \mathrm{CH}_{3}$ or $\mathrm{CF}_{3}, \mathrm{~L}=\mathrm{NH}_{3}$ or pyridine, and $n=0,1$ or 2 . It correctly generates the distorted tetrahedral coordination of the uncapped $\left[\mathrm{Zn}_{2}\left(\mathrm{O}_{2} \mathrm{CR}\right)_{4}\right]$ species in their ground states as well as giving reasonable structures and energies for the higher symmetry $\mathrm{D}_{4 \mathrm{~h}}$ transition state conformations. The zinc-ligand Morse function reference distance, $r_{0}$, is further refined against 30 complexes located in the Cambridge Structural Database and this FF is applied to pore models of the flexible metal-organic framework (MOF) $\left[\mathrm{Zn}(\mathrm{bdc})_{2}(\mathrm{dabco})\right]_{\mathrm{n}}(\mathrm{bdc}=1,4$-benzendicarboxylate; dabco $=1,4$-diazabicyclo(2.2.2)octane). A single pore model reproduces the unit cell of the evacuated MOF system while a $3 \times 3$ grid model is necessary to provide good agreement with the observed pronounced structural changes upon adsorption of either dimethylformamide or benzene.
\end{abstract}

Electronic supplementary material The online version of this article (doi:10.1007/s00894-016-2949-5) contains supplementary material, which is available to authorized users.

Robert J. Deeth

r.j.deeth@warwick.ac.uk

1 Inorganic Computational Chemistry Group, University of Warwick, Coventry CV4 7AL, UK

2 Present address: School of Chemistry, Joseph Black Building, David Brewster Road, Edinburgh, Scotland EH9 3FJ, UK
Keywords Force field · Metal organic framework $\cdot$ Molecular dynamics $\cdot$ Molecular mechanics $\cdot$ Transition metal complex

\section{Introduction}

Metal organic frameworks (MOFs) are porous materials with a remarkable range of potential applications [1-3]. The framework comprises combinations of secondary building units (SBUs) connected by linkers which can generate a remarkable array of 3-dimensional networks. The SBUs are transition metal complexes or clusters while the linkers are typically organic carboxylates often in combination with polytopic nitrogen-donor 'pillar' ligands such as, for example, 1,4diazabicyclo(2.2.2)octane (dabco) or pyrazine.

While many MOFs have relatively rigid frameworks which therefore define a fixed pore size, other MOFs display a degree of flexibility or 'breathing' $[4,5]$. The pore size and/or shape changes as a function of adsorbate offering exciting possibilities for using these materials in separations [6-9] and sensing $[10,11]$.

Some flexible MOFs contain a paddle-wheel SBU. The paddle-wheel motif is a TM dimer bridged by three or four carboxylate units. In combination with linear linkers, the latter generates planar $\left[\mathrm{M}_{2} \mathrm{~L}_{2}\right]_{\mathrm{n}}$ grids which can be interconnected by ditopic pillars like dabco to generate a 3-D framework. The classic example is the MOF $\left[\mathrm{Zn}_{2}(\mathrm{bdc})_{2}(\mathrm{dabco})\right]_{\mathrm{n}}(\mathrm{bdc}=1,4-$ benzenedicarboxylate) which displays a remarkable degree of flexibility depending on the adsorbate ${ }_{[12]}$.

As synthesized, the $\left[\mathrm{Zn}_{2}(\mathrm{bdc})_{2}(\mathrm{dabco})\right]_{\mathrm{n}}$ pore contains one water and four dimethylformamide (dmf) molecules. The framework-adsorbate interactions lead to a pronounced bending deformation of the pore bdc edges (Fig. 1, left) but this disappears on evacuation leaving a more regular cuboidal pore (Fig. 1, middle) which further distorts (and contracts) to a 
Fig. 1 Pore framework structures for $\left[\mathrm{Zn}_{2}(\mathrm{bdc})_{2}(\mathrm{dabco})\right]_{\mathrm{n}}$ derived from published CIF files [12] Hydrogens and encapsulated solvent removed. Dabco and carboxylate disorder as per CIF file

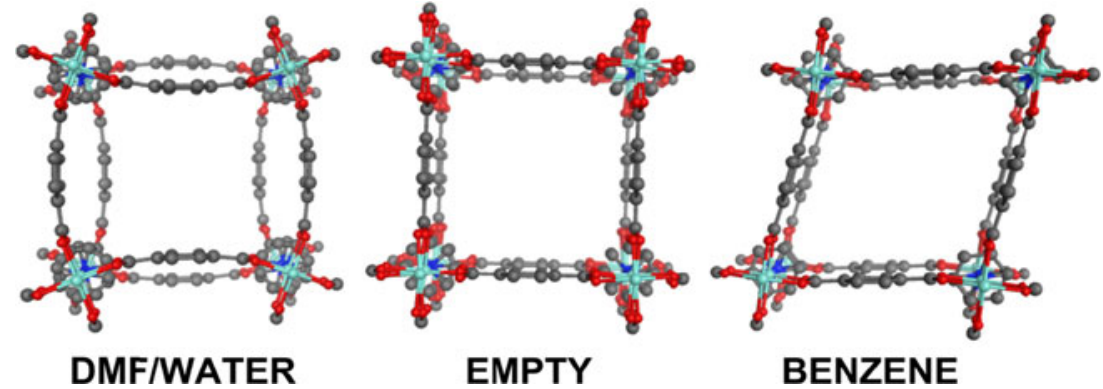

rhombohedral structure upon adsorption of benzene (Fig. 1, right).

Computer modeling of MOFs can provide important atomic level insights into their structures and properties but the structural changes of the type shown in Fig. 1 are computationally challenging and demand a sophisticated theoretical method [13]. Quantum chemical (QC) approaches such as density functional theory (DFT) are fairly general, reasonably accurate and give satisfactory results for a wide range of transition-metal systems. However, MOFs are relatively large and QC is computationally expensive, especially if dynamical properties are of interest [13]. Nevertheless, ab initio molecular dynamics (AIMD) is possible and has been applied, for example, to the breathing of MIL-53(Sc) [14]. Despite the short simulation times of only a few ps, impressive agreement with experiment was obtained. Given its general applicability, we can anticipate many more QC and AIMD studies in the future. Meanwhile, modeling very large systems for a long time, or carrying out virtual high throughput screening,[15] remains the province of classical simulation techniques.

Classical methods such as grand canonical Monte Carlo (GCMC) have been widely employed to model the thermodynamics of adsorbate-MOF interactions $[16,17]$ but these generally assume a fixed framework which may be inappropriate in the case of flexible MOFs. The alternative is to consider fully atomistic molecular mechanics/molecular dynamics (MM/MD) simulations.

The critical feature of $\mathrm{MM} / \mathrm{MD}$ is the underlying force field (FF). Generic FFs like UFF [18] and Dreiding [19] can be applied to MOFs but their performance may be of limited accuracy [20]. Thus, while a universal force field is attractive, it is also an extremely challenging undertaking and most of the recent FF development targeted at MOF systems has involved so-called 'first principles' parameterization wherein the FF parameters are derived from quantum-chemically-generated training data [21-23]. Bespoke FFs designed for MOFs such as BTW-FF[24], MOF-FF [22], and UFF4MOF [25] should give better accuracy but perhaps at the expense of development time and transferability-i.e., the FF may only work well for the subset of MOFs on which they are trained. In the case of the flexible MOF $\mathrm{NH}_{2}-\mathrm{MIL}-53(\mathrm{Al})$, Garcia-Perez et al. even argue [26] that a fully flexible FF is unnecessary and that a combination of rigid FF combined with some judicious experimental work is sufficient to predict adsoprtion and diffusion of $\mathrm{CO}_{2}$ and methane through this material.

In our work deriving accurate FFs targeted at specific transition-metal/ligand combinations, we have used both experimental and/or quantum chemical data [27-35]. Here, we focus on a particular class of flexible MOFs which incorporate the four-bladed zinc paddlewheel (ZPW) motif and construct a new, specialized valence FF, ZPW-FF, based on molecular ZPW complexes which then automatically captures the types of structural change displayed by $\left[\mathrm{Zn}_{2}(\mathrm{bdc})_{2}(\text { dabco })\right]_{\mathrm{n}}$ as a function of adsorbate. To achieve this, we consider the DFTcalculated chemistry of simple model ZPW systems including those for which there are no experimental data such as the uncapped 'bare' $\mathrm{Zn}_{2}$ (carboxylate) $)_{4}$ unit. The latter has either not been explicitly included in the FF development (BTW-FF [24] and MOF-FF [22]) or the structure employed was not the ground state (UFF4MOF [25]). Our new FF is thus based on a consistent set of theoretical data but is then further refined using the structural chemistry of experimentally characterized, single-ZPW systems to reduce the systematic errors from our chosen DFT protocol. The focus on the local coordination environment of the zinc centers gives the ZPW-FF an unprecedented ability to reproduce subtle variations in bond lengths and bond angles and provides DFT-like accuracy at a small fraction of the computational cost. The good accuracy extends to modeling MOFs and we demonstrate that the structural changes observed for $\left[\mathrm{Zn}_{2}(\mathrm{bdc})_{2}(\text { dabco })\right]_{\mathrm{n}}$ can be successfully reproduced using non-periodic models of its pores. Significantly, the ZPW-FF is based on single ZPW systems so that the subsequent flexibility of the multiple-ZPW pore models emerges as a natural property predicted by ZPW-FF.

\section{Theoretical methods}

All the DFT calculations reported here used the ORCA suite version 3.0.1 [36]. The general protocol employed the BeckePerdew BP86 functional [37, 38] with Ahlrichs' def2-SVP basis sets [39]. Condensed phase effects [40] were accounted for using the conductor like screening model (COSMO) [41-43] as implemented in ORCA with water as the solvent. 
Molecular mechanics optimizations used DommiMOE [44], our extension of the 2011 version of the molecular operating environment (MOE) [45]. The as-distributed Merck molecular force field, MMFF94, (mmff94x.ff) was augmented with additional Zn-L-A angle-bending terms and Zn-L-A-B torsional terms. Ligand field molecular mechanics (LFMM) parameters were defined for the zinc coordination [46]. Zn-L interactions were described via Morse functions and the explicit angle bending terms were replaced by a pure ligand-ligand repulsion term of the form $A_{L L} / d^{n}$. For these $\mathrm{d}^{10} \mathrm{Zn}^{2+}$, there is no ligand field stabilization energy and hence all angular overlap model parameters and spin-pairing terms were set to zero. The MOE and LFMM parameter files and partial-charge-setting scripts are included in the supporting information and are also available from the authors (RJD) upon request [47]. Unless otherwise noted, electrostatic interactions employ a distancedependent dielectric term with a damped cut-off starting at $8 \AA$ going to zero at $10 \AA$.

A typical NVT ensemble molecular dynamics annealing protocol was as follows: starting $\mathrm{T}=50 \mathrm{~K}$; heat to $330 \mathrm{~K}$ in $10 \mathrm{ps}$, hold for $10 \mathrm{ps}$, cool to $0 \mathrm{~K}$ in $10 \mathrm{ps}$. The Nosé-PoincaréAndersen algorithm was employed with a 2 fs time step. Bond lengths to $\mathrm{H}$ atoms were frozen. A $0.1 \mathrm{fs}$ temperature damping constant was used with configurations sampled every $0.5 \mathrm{ps}$.

\section{Results and discussion}

A good force field for coordination compounds relies on a diverse set of training data $[48,49]$. Our previous experience with $\mathrm{Cu}(\mathrm{II}) \mathrm{FFs}$ shows that the inherent 'plasticity' of the Jahn-Teller active $\mathrm{d}^{9}$ center yields sufficient diversity that an accurate FF can be constructed using structural data derived solely from experimental X-ray diffraction studies [50]. This is not quite the case for ZPW systems which, at first sight, all seem remarkably similar.

The Cambridge Structural Database (CSD) is a rich source of experimental structural data. Our initial searches were restricted to a central ZPW motif such that none of the Zn$\mathrm{O}$ (carboxylate) bonds were coded as 'polymeric'. This search thus excludes the majority of (but not all) ZPW MOFs in favor of compounds with isolated, molecular ZPW units and yielded 77 ZPW structures. The Zn-O(carboxylate) distances do not vary very much and average at $2.04 \AA$ with a standard deviation of $\sim 0.03 \AA$.

Starting with the extremes, the shortest $\mathrm{Zn}-\mathrm{O}$ (carboxylate) bond length is reported to be $1.88 \AA$ for catena-(tetrakis $\left(\mu_{6}-1\right.$, 1',1"-(1,3,5-triazine-2,4,6-triyl)tripiperidine-4-carboxylic acid)-hexaaqua-hexa-zinc(II) pyridine dimethyl sulfoxide solvate (CSD refcode WUHHEN) [51]. Although this compound is actually a MOF, the unit cell is sufficiently large to accommodate complete ZPW units and hence pass our test of not having polymeric $\mathrm{Zn}-\mathrm{O}$ contacts. However, there are a number of anomalous structural features as illustrated in Fig. 2.

The carboxylates are oddly coordinated, the internal C-O distances are very asymmetric and the hydrogen attached to the adjoining carbon atom is not reported in the CIF file. Given that there is significant disorder of incorporated solvent molecules and the overall $\mathrm{R}$ factor for the refinement is relatively high ( $8.4 \%)$, the short $\mathrm{Zn}-\mathrm{O}$ contact seemed anomalous to us. In any event, this system has water apical ligands and this paper focuses on apical $\mathrm{N}$ donor. The extension of ZPW$\mathrm{FF}$ to apical oxygen donors and the anomalous $\mathrm{X}$-ray structure of WUHHEN will be the subject of a future publication.

The longest $\mathrm{Zn}-\mathrm{O}$ bond length in the set of $2.13 \AA$ in (tetrakis $\left(\mu_{2}\right.$-benzoato)-bis(pyridine-4-carbaldehyde oxime)di-zinc(II) (TUFLOW), has a ready experimental interpretation in terms of the intermolecular H-bond between the carboxylate oxygen and the 4-pyridyl-oxime ligand of a neighboring ZPW complex as highlighted in magenta in Fig. 3. This example hints at the sensitivity of the ZPW structure which appears to be relatively easy to distort. This should also provide an exacting test of a force field's ability to model intermolecular interactions accurately. However, the initial FF development focuses more on intra-molecular interactions.

Although the overall metal coordination in ZPW system is invariant- every example in the CSD shows five-coordinate $\mathrm{Zn}$ centers-there are some subtle variations which a FF should deal with. Given the geometric constraints of the paddle-wheel motif, the local zinc coordination is approximately square pyramidal, especially with nitrogen in the apical position. However, in the absence of electronic effects, as would be expected here for $\mathrm{d}^{10} \mathrm{Zn}^{\mathrm{II}}$ species, five-coordinate complexes should prefer to be trigonal bipyramidal. In addition, pentacoordinate species are well known to be quite flexible and readily undergo Berry pseudorotations. The zinc sites in ZPWs are thus inherently unstable from a mechanical

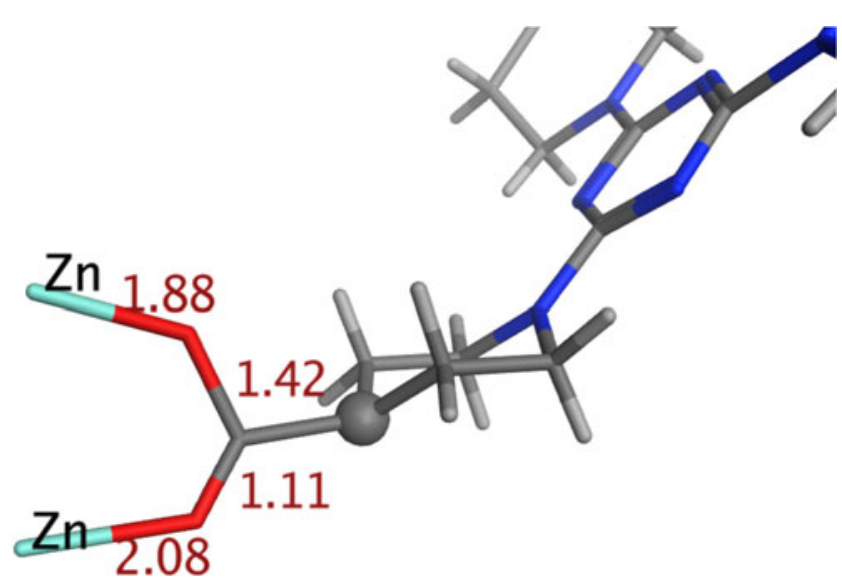

Fig. 2 Local detail of carboxylate coordination in WUHHEN. Bond lengths $(\AA)$ shown in dark red. The upper $\mathrm{Zn}-\mathrm{O}$ contact is anomalously short, the carboxylate $\mathrm{C}-\mathrm{O}$ bonds too asymmetric and one hydrogen is missing off the highlighted carbon (gray sphere) 


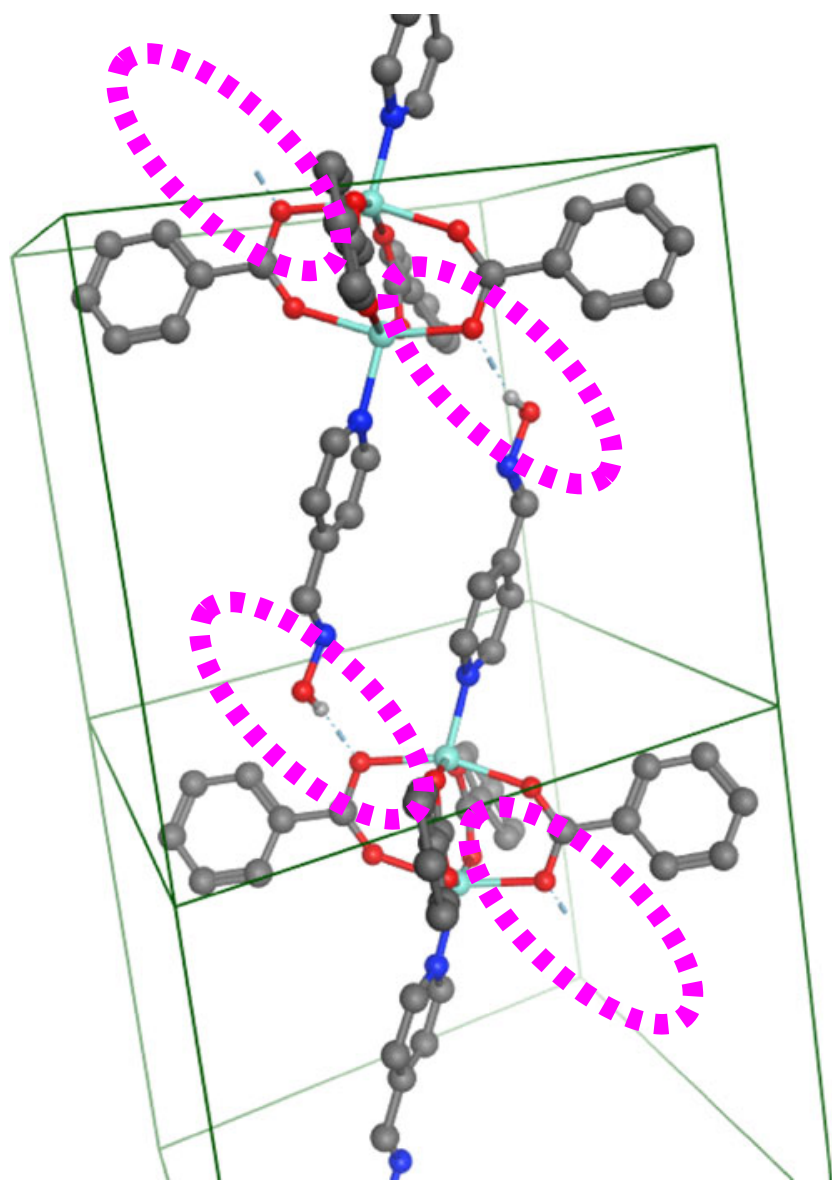

Fig. 3 Packing detail for TUFLOW showing intermolecular H-bond contacts (dotted magenta oval) responsible for the long $\mathrm{Zn}-\mathrm{O}$ distance. (The extra connecting molecules top right and bottom left are omitted for clarity as are all the $\mathrm{H}$ atoms bar those involved in the $\mathrm{H}$-bond)

perspective and this manifests itself as a subtle sensitivity of the ZPW to intra- and inter-molecular interactions.

A number of distortions from a regular ZPW structure can be conceived (Fig. 4): (i) a twist around the $\mathrm{Zn}-\mathrm{Zn}$ vector, (ii) a shear of one $\mathrm{ZnO}_{4} \mathrm{~L}$ unit relative to the other, and (iii) a sliding motion of a pair of trans-related carboxylates parallel to the $\mathrm{Zn}-\mathrm{Zn}$ vector toward one of the zinc centers while the other two carboxylates move in the opposite direction. This makes both $\mathrm{Zn}$ geometries more trigonal bipyramidal.

Examples of twisted and sheared structures can readily be found in the CSD data but there are apparently no good experimental examples of a pronounced sliding distortion at least with capping $\mathrm{N}$ donor ligands. However, such structures can be generated computationally (vide infra). DFT is the obvious method and we optimized the structures of a number of ZPW systems both to test the viability of the DFT protocol (BP86/SVP/COSMO(water)) as well as to investigate the source of any apparent distortions in the crystallographic structures. Our choice of functional is based on previous experience [52] plus we note that it was also used for UFF4MOF [25] although the latter employed the ADF program [53],

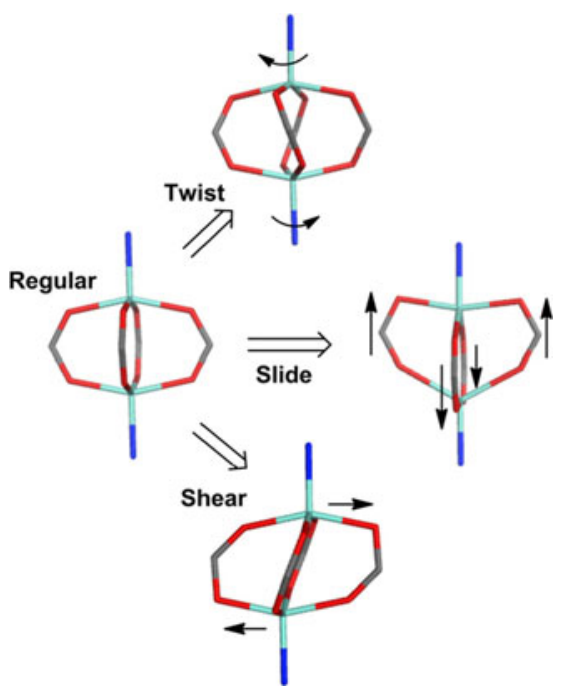

Fig. 4 Schematic idealizations of possible distortions of a regular zinc paddlewheel structure

triple- $\zeta$ STO basis sets and ZORA [54] relativisitic corrections. In any event, a recent benchmarking study suggests that comparable results can be obtained for a wide range of functionals [55] so the particular choice made here is not expected to be especially significant.

As expected, the BP86/DFT/COSMO protocol generally reproduces the experimental structures very well (see Supporting information for overlaid structures). However, the averaged data in Table 1 also show that the chosen DFT methodology systematically overestimates the zinc-ligand distances by around 0.03 to $0.05 \AA$. The FF will be corrected for this error subsequently.

The initial FF parameterization is based exclusively on first-principles DFT data. A previous DFT study of zinc and copper paddlewheel systems revealed some interesting structural features [56]. In particular, the ground state for an uncapped ZPW displays a large sliding distortion of $\mathrm{D}_{2 \mathrm{~d}}$ symmetry consistent with the four-coordinate $\mathrm{Zn}$ centers trying to adopt a tetrahedral geometry. The higher symmetry $\mathrm{D}_{4 \mathrm{~h}}$ structure is a transition state. To our knowledge, this feature has not been modeled with any previous FFs for ZPW systems although MOF-FF is formulated in a way which may be able to reproduce the correct ground state [22]. However, the use of an explicit angle-bending term in MOF-FF, even though based on a Fourier series which generates more than one reference angle [57], is not as flexible as the current ZPW-FF approach which has no explicitly angle-bending term at the metal centers and uses instead a 1-3 interaction potential exclusively $[58,59]$. Our experience suggests, especially for coordination numbers greater than four, that this should be a better approach compared to methods like UFF4MOF [25] and BTW-FF [24] which have parameters which enforce a particular coordination geometry for a given coordination number. Moreover, the UFF4MOF implementation only considers the 
Table 1 Comparison of experimental and calculated bond lengths $(\AA)$ for the complexes shown in Fig. 5. The Zn-O(CBX) entry is the averaged Zn$\mathrm{O}$ (carboxylate) distance. $\mathrm{Zn}-\mathrm{L}$ refers to the bond to the capping group

\begin{tabular}{|c|c|c|c|c|c|c|c|c|c|c|c|c|c|c|}
\hline \multirow[t]{2}{*}{ CSD Refcode } & \multicolumn{2}{|c|}{ AGAHEV } & \multicolumn{2}{|c|}{ AZOGOL } & \multicolumn{2}{|c|}{ ВОНХОМ } & \multicolumn{2}{|c|}{ DOYZIA } & \multicolumn{2}{|c|}{ EBEPEG } & \multicolumn{2}{|c|}{ HOPTUC } & \multicolumn{2}{|c|}{ KIKXIM } \\
\hline & X-ray & DFT & $\mathrm{X}$-ray & DFT & X-ray & DFT & $\mathrm{X}$-ray & DFT & $\mathrm{X}$-ray & DFT & $\mathrm{X}$-ray & DFT & $\mathrm{X}$-ray & DFT \\
\hline $\mathrm{Zn}-\mathrm{Zn}$ & 3.23 & 3.23 & 3.19 & 3.22 & 2.98 & 2.93 & 2.98 & 2.96 & 2.93 & 2.96 & 2.98 & 2.83 & 2.97 & 3.05 \\
\hline $\mathrm{Zn}-\mathrm{O}(\mathrm{CBX})$ & 2.05 & 2.09 & 2.07 & 2.10 & 2.04 & 2.07 & 2.04 & 2.07 & 2.03 & 2.07 & 2.04 & 2.06 & 2.03 & 2.08 \\
\hline $\mathrm{Zn}-\mathrm{L}$ & 1.92 & 1.97 & 2.07 & 2.07 & 2.04 & 2.07 & 2.06 & 2.09 & 1.99 & 2.02 & 1.99 & 2.06 & 2.09 & 2.15 \\
\hline$\Delta(\mathrm{Zn}-\mathrm{Zn})$ & & 0.00 & & -0.03 & & 0.05 & & 0.02 & & -0.03 & & 0.16 & & -0.07 \\
\hline$\Delta(\mathrm{Zn}-\mathrm{O}(\mathrm{CBX}))$ & & -0.04 & & -0.03 & & -0.03 & & -0.03 & & -0.04 & & -0.02 & & -0.05 \\
\hline$\Delta(\mathrm{Zn}-\mathrm{L})$ & & -0.05 & & 0.00 & & -0.03 & & -0.02 & & -0.03 & & -0.07 & & -0.05 \\
\hline
\end{tabular}

higher symmetry transition state structure for the 'bare' system plus an explicit $\mathrm{Zn}-\mathrm{Zn}$ bond is employed which is not physically reasonable but required to generate better structures.

Experimentally, ZPWs all appear to have pentacoordinate metal centers so it is not surprising that no-one has considered a FF for four-coordinate zinc centers. However, this is quite significant for $\mathrm{Cu}$ paddlewheel analogues which often display 'naked' metal centers plus it also goes toward the inherent flexibility of the MOF.

Given that the vast majority of ZPW MOFs have either pyridyl $\mathrm{sp}^{2}$ or amine $\mathrm{sp}^{3}$ nitrogen capping ligands, the required MMFF94 ligand parameter atom types are NPYD, N, and OX, the latter referring to carboxylate oxygens. Three carboxylates are considered for the basic training species: formate, acetate and trifluoroacetate $\left(\mathrm{R}=\mathrm{H}, \mathrm{CH}_{3}\right.$ or $\left.\mathrm{CF}_{3}\right)$ with zero, one or two capping groups, $\mathrm{L}$, which are either pyridine (py) or ammonia $\left(\mathrm{NH}_{3}\right)$ (Fig. 6). There are thus five complexes for each carboxylate with each species having a general formula $\mathrm{ZnPR} . \mathrm{nL}$, where $\mathrm{ZnP}$ represents the $\mathrm{Zn}_{2}\left(\mathrm{O}_{2} \mathrm{C}\right)_{4}$ core of the ZPW. The choice of carboxylates was motivated from a consideration of $\mathrm{pK}_{\mathrm{a}}$ values. The $\mathrm{pK}_{\mathrm{a}}$ of acetic acid (4.76) is among the higher values with trifluoroacetic acid $(0.23)$ the lowest. Formate and benzoic acids are intermediate and hence the chosen acids span the relevant $\mathrm{pK}_{\mathrm{a}}$ range. Benzoic acid is not in the training set but occurs frequently in the validation set (vide infra).

Partial atomic charges were modified from the previously published FF for Mn(II) species [60] and are based on superimposing the change in Mulliken charges between uncoordinated and coordinated ligands onto the existing bond charge increment scheme from the Mn(II) FF. The new values are listed in Table 2 along with the standard MMFF94 values for comparison.

Although there are no d-electron effects for $\mathrm{Zn}(\mathrm{II})$, we use the ligand field molecular mechanics (LFMM) method [46, 58] as implemented in DommiMOE,[44] our extended version of the molecular operating environment (MOE) [61]. LFMM parameters for M-L bond stretching (Morse function $r_{0}$ and $\alpha$ ), ligand-ligand repulsion $\left(A_{\mathrm{LL}}\right), \mathrm{Zn}$-L-A angle bending $\left(\theta_{0}\right.$ and $\left.k_{\theta}\right)$, and Zn-L-A-B torsional twisting $\left(V_{2}\right.$ which favors torsions of 0 and $180^{\circ}$ ) were manually optimized to minimize the rmsd in $\mathrm{Zn}-\mathrm{L}$ bonds and heavy-atom (i.e., nonhydrogen) overlays.

The resulting ZPW-FF (see Supporting information) reproduces DFT very accurately (see Fig. 7). The overall rmsd in $\mathrm{Zn}-\mathrm{L}$ distances for all 15 systems is $0.02 \AA$ with the highest value for any one complex being only $0.03 \AA$. The largest individual error in a single $\mathrm{Zn}-\mathrm{L}$ bond length is $0.046 \AA$ for
Fig. 5 A selection of entries from the CSD used to validate the DFT protocol

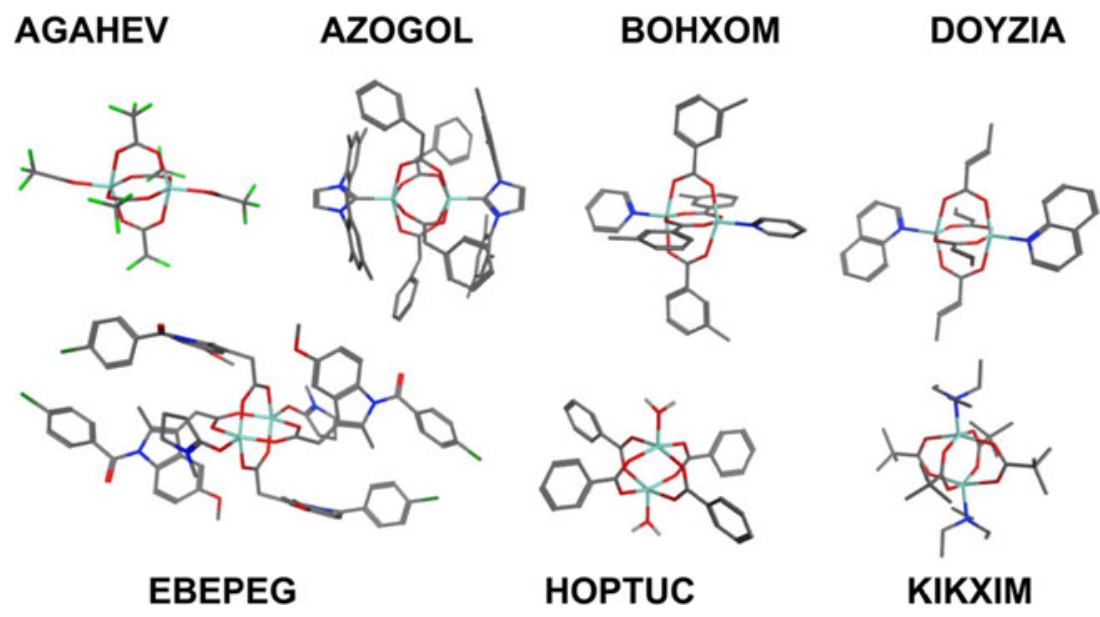




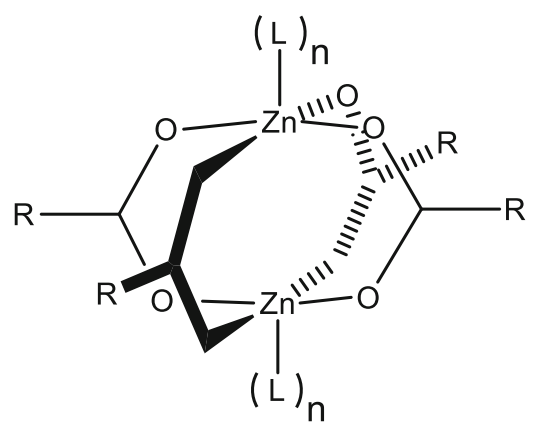

$\mathrm{L}=\mathrm{NH}_{3}$, py

$\mathrm{n}=0,1$

$\mathrm{R}=\mathrm{H}, \mathrm{CH}_{3}, \mathrm{CF}_{3}$

Fig. 6 Schematic representation of the ZnPR.nL systems used for initial training

two of the $\mathrm{Zn}-\mathrm{O}$ contacts on the uncapped end of $\mathrm{ZnPCF}_{3} \cdot \mathrm{NH}_{3}$.

The reproduction of the angular geometries is also excellent as evidenced by the overall average rmsd for heavy atom (i.e., non-hydrogen) overlays of only $0.16 \AA$. The FF successfully captures the broad variation in zinc coordination as well as a number of subtle structural features as illustrated for the formate species in Fig. 7. MM tends to be a little more 'tetrahedral' than DFT, but the detailed variation in bond lengths and angles is remarkably well reproduced. This is especially apparent for the lower symmetry species where, for example, the $\mathrm{Zn}-\mathrm{O}$ distances can vary substantially. In $\left[\mathrm{Zn}_{2}\left(\mathrm{O}_{2} \mathrm{CH}\right)_{4}\right]$, there are two symmetry independent types of oxygen donor with coordination distances differing by $0.16 \AA$ in the DFT optimization. The ZPW-FF predicts a difference of $0.18 \AA$. The mono-capped species have an approximately trigonal bipyramidal five-coordinate zinc center in conjunction with a flattened tetrahedral four-coordinate center. Again, the detailed agreement between DFT and ZPW-FF for both bondlength and bond angle variations is excellent.

Table 2 New partial atomic charges for MMFF94 implementation of ZPW-FF. Standard MMFF94 charges in parentheses

\begin{tabular}{|c|c|c|}
\hline Atom type & Environment & $\begin{array}{l}\text { New partial charges } \\
\text { (standard MMFF94 charge) }\end{array}$ \\
\hline $\mathrm{OX}$ & All & $-0.72(-0.90)$ \\
\hline $\mathrm{N}$ & $\mathrm{NH}_{3}$ & $-0.90(-1.08)$ \\
\hline $\mathrm{HN}$ & $\mathrm{NH}_{3}$ & $0.43(0.36)$ \\
\hline $\mathrm{N}$ & $\mathrm{N}\left(\mathrm{Csp}^{3}\right)_{3}$ & $-0.63(-0.81)$ \\
\hline $\mathrm{C}$ & Adjacent to $\mathrm{N}\left(\mathrm{Csp}^{3}\right)_{3}$ & $0.34(0.27)$ \\
\hline NPYD & All & $-0.42(-0.62)$ \\
\hline Car & Adjacent to NPYD & $0.23(0.16)$ \\
\hline $\mathrm{HC}$ & H-C-NPYD & $0.23(0.15)$ \\
\hline $\mathrm{CO} 2 \mathrm{M}$ & $\mathrm{C}$ of formate & $0.74(1.02)$ \\
\hline $\mathrm{CO} 2 \mathrm{M}$ & $\mathrm{C}$ connected to $\mathrm{sp}^{3}$ carbon & $0.626(0.906)$ \\
\hline $\mathrm{Zn}+2$ & four carboxylates & 1.84 \\
\hline $\mathrm{Zn}+2$ & four carboxylates $+\mathrm{N}$ & 1.45 \\
\hline $\mathrm{Zn}+2$ & four carboxylates + NPYD & 1.34 \\
\hline
\end{tabular}

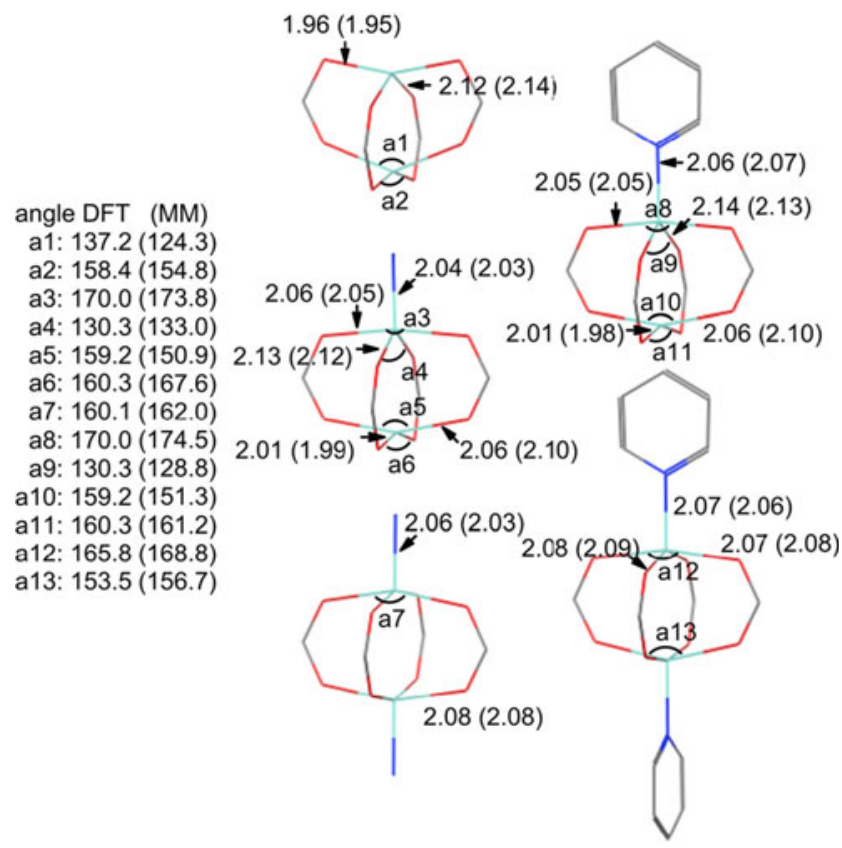

Fig. 7 Comparison of optimized DFT and MM (in parentheses) structural parameters for $\mathrm{ZnPH} . \mathrm{nL},\left(n=0,1,2 ; \mathrm{L}=\mathrm{NH}_{3}\right.$, py). Only unique $\mathrm{Zn}-\mathrm{L}$ and $\mathrm{L}-\mathrm{Zn}-\mathrm{L}$ data shown. Distances in $\AA$ and angles in degrees. Hydrogens omitted for clarity. Depicted structures are from DFT-optimized coordinates

Using the same ZPW-FF parameters, the structures and relative energies of the transition states for the uncapped species can be compared (Table 3). The structures were optimized using a simple in-house Newton-Raphson code which follows the largest negative eigenvalue. Good starting points for the TS search are needed and these can be conveniently generated by deleting the two capping groups from a ZnPR.2 L system.

The ZPW-FF barriers are about $30 \%$ lower than those from DFT but the overall agreement is satisfactory.

Recalling the systematic errors between experimental and DFT-optimized complexes, the current FF was retuned using experimental X-ray structural data of $32 \mathrm{ZPW}$ complexes comprising 30 unique ligand combinations (Fig. 8). The refinement was restricted to minor adjustments of Morse $r_{0}$ values to minimize the rmsd $\mathrm{Zn}-\mathrm{L}$ deviations (see Supporting information). The data in Table 4 and in the Supporting information show that the adjusted FF delivers good structural accuracy. The average deviation in zincligand distances is $0.04 \AA$ (Table 4) while $92 \%$ of the individual deviations for all $\mathrm{Zn}-\mathrm{L}$ distances are less than $0.05 \AA$ and only $1 \%$ of all bond length deviations is larger than $0.1 \AA$ with the largest error being $0.137 \AA$ for XAYKOY (see Supporting information). The overall shapes of the complexes are well reproduced with generally small heavy atom overlay rmsds (Table 4). The latter in particular will be subject to crystal packing influences so we cannot expect (nor want) exact agreement. However, overall, the results give us some 
Table 3 Calculated $\mathrm{Zn}-\mathrm{O}$ bond lengths $(\AA)$, activation energies $\left(\mathrm{kcal} \mathrm{mol}^{-1}\right)$ and transition-state frequencies $\left(\mathrm{cm}^{-1}\right)$ for $\mathrm{ZnPR}$ transition state systems, $\mathrm{R}=\mathrm{H}$, $\mathrm{CH}_{3}$, and $\mathrm{CF}_{3}$

\begin{tabular}{lllccccc}
\hline $\mathrm{R}$ & $\mathrm{r}^{\ddagger}(\mathrm{Zn}-\mathrm{O}): \mathrm{DFT}$ & $\mathrm{r}^{\ddagger}(\mathrm{Zn}-\mathrm{O}): \mathrm{MM}$ & $\Delta \mathrm{E}^{\ddagger}: \mathrm{DFT}^{1}$ & $\Delta \mathrm{G}^{\ddagger}: \mathrm{DFT}^{2}$ & $\Delta \mathrm{E}^{\ddagger}: \mathrm{MM}$ & $\nu^{\ddagger}: \mathrm{DFT}$ & $\nu^{\ddagger}: \mathrm{MM}$ \\
\hline $\mathrm{H}$ & 2.04 & 2.06 & 9.4 & 9.2 & 6.3 & -87 & -62 \\
$\mathrm{CH}_{3}$ & 2.03 & 2.07 & 10.8 & 10.3 & 7.6 & -84 & -57 \\
$\mathrm{CF}_{3}$ & 2.04 & 2.06 & 7.4 & 7.9 & 5.3 & -60 & -44 \\
\hline
\end{tabular}

${ }^{a}$ Raw DFT electronic energy difference

${ }^{\mathrm{b}}$ Free energy difference computed using standard statistical mechanics methods implemented in ORCA, $P=1 \mathrm{~atm}, \mathrm{~T}=298 \mathrm{~K}$

confidence to carry on to modeling more complicated systems such as MOFs which contain multiple, interconnected ZPW units.

Flexible MOFs Our preliminary investigations into flexible MOF materials focuses on models of individual pores and small grids of pores for $\left[\mathrm{Zn}_{2}(\mathrm{bdc})_{2}(\mathrm{dabco})\right]_{\mathrm{n}}$. We are motivated by the desire to explore the intimate inter- and intramolecular interactions both within the framework and between the framework and adsorbed species to assess whether the flexible structures are an inherent feature of the pore. We recognize that many fundamental mechanical, thermal, and dielectric properties will not be available [24] and that care will be required when translating our findings into systems which, in reality, are fully periodic. Nevertheless, we believe the results reported below are significant both in their own right and in terms of a future development of a fully periodic implementation in our DL_POLY_LF platform [62]. Meanwhile, we consider the various ZPW systems highlighted in Fig. 1.

A model for a single pore was constructed from the crystallographic CIF file. It comprises eight ZPW units at the pore corners with dabco pillars. Corner carboxylates are capped with hydrogen while corner zinc centers are capped with $\mathrm{Me}_{3} \mathrm{~N}$ groups. The $\mathrm{X}$-ray structure shows significant disorder which results in the dabco groups not having threefold symmetry and the orientations of the dmf carbonyl oxygens is ambiguous. We have selected an arrangement such that the carbonyl oxygen of one dmf is directed toward the $\mathrm{HC}(\mathrm{O})$ hydrogen of a neighboring dmf. Water molecules are also identified in the crystal structure but their position at the face of the pore would make them susceptible to effects from adjoining pores. Also, preliminary calculations showed that they have strongly directional $\mathrm{H}$-bonding effects and tend to migrate to the nearest ZPW unit causing significant local distortions. This is one of the shortcomings of using an aperiodic system and the water molecules have therefore not been inclued in the subsequent MM optimizations. Also, given that there are now multiple ZPW units as apposed to the single ZPW systems considered so far, the standard $8 \AA / 10 \AA$ electrostatic cut-offs are disabled and the default distancedependent $\left(1 / \varepsilon \mathrm{r}^{2}\right)$ dielectric model for electrostatics is replaced by the softer reaction field implemented in MOE (see Supporting information). Both these changes have minimal effects on either the training systems or the refinement/ validation set. The final single-pore model is shown in Fig. 9.

MM optimizations of the single-pore model with four $\mathrm{dmf}$ molecules incorporated display a definite curvature of the pore edges (Fig. 10, middle top). Removal of the dmf molecules leads to a regular 'cuboidal' geometry while replacement of the $\mathrm{dmf}$ by benzene molecules gives a pronounced rhombohedral distortion. The MM-optimized pores are thus consistent with experiment but, apart from the vacant pore, the calculated distortions are underestimated. In practice, each pore vertex is connected to another pore and it is probably not surprising that a single-pore model is inadequate. Hence, we extended the model to a $3 \times 3$ grid of nine pores so that the central one is connected on all sides.

As shown on the right side of Fig. 10, this leads to substantially better agreement with the reported $\mathrm{X}$-ray structural data. The calculated unit cell dimensions of $\mathrm{a}=11.0 \AA$ and $\mathrm{c}=9.6 \AA$ for the empty pore are virtually identical to the experimental values of 10.9288(15) and 9.6084(12) while for the dmf adsorbate system, the dihedral twist between two ZPW groups connected by a dabco pillar increases from $\sim 9^{\circ}$ for the 1-pore model to $\sim 30^{\circ}$ for the 9-pore model compared to the experimental value of $40^{\circ}$. This twist is accommodated in the modeling largely by a hinge movement along the O-O vector of the carboxylates which keeps the bdc unit flat as opposed to the $\mathrm{X}$-ray structure which shows a smoother curved profile of the bdc bridge. For the benzene-solvated system, the acute angle formed from, for example, the top three zinc centers at the vertices of the pore changes from $81^{\circ}$ for the 1-pore model to $75^{\circ}$ for the 9 -pore model compared to $77^{\circ}$ from the X-ray structure.

The current FF thus appears to capture the breathing modes of this particular flexible MOF quite well using an aperiodic model system which we believe is a significant achievement given that only single-ZPW unit systems were employed in its construction. However, part of this success is undoubtedly due to the pillared structure, which means we only need to consider a single layer, plus the positions of the solvent molecules have been taken from experiment. A greater test would be to see whether the number and position of solvent molecules within the pore might be predicted.

We start with benzene since it is more symmetrical than $\mathrm{dmf}$ and the experimental structure is not disordered. A single benzene molecule was placed at the center of an empty 1-pore 
Fig. 8 Structural diagrams of ligands for the ZPW systems listed by CSD refcode in Table 4. Only unique combinations displayed. (NEHZUV and NEHHUV01 are the same compound while INIBAJ and QETGAY have the same ligand set)

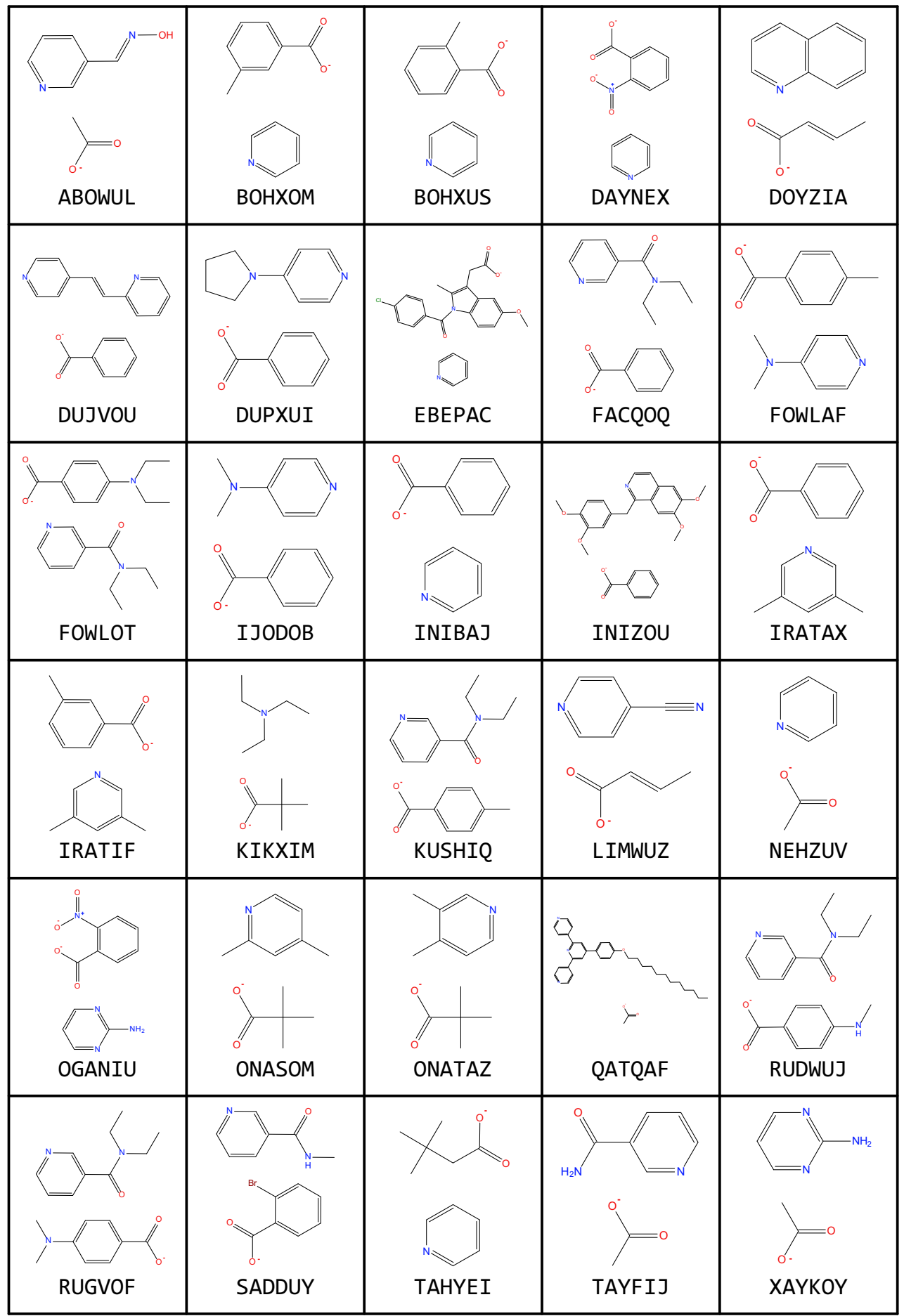

model and energy minimized. It spontaneously migrated to the methylene units of a dabco pillar. Adding a second benzene to this structure, again placed at the center of the pore, and energy minimizing led to the second benzene spontaneously migrating to the opposite dabco pillar. Adding a third benzene to this model, which clearly generates a highly strained starting point, spontaneously leads to the third benzene migrating to the pore surface to form van der Waals contacts with bdc linker aromatic rings. So far, the benzene groups have positioned themselves exactly where the experiment suggests and there is a clear visual indication of where the next benzene should go. However, following the same computational protocol, energy minimization once a fourth benzene is added leads to two results. Sometimes, the fourth 
Table 4 Performance of ZPW-FF for molecules shown in Fig. 8. Column 1 CSD refcodes. Column 2, root mean square deviation for $\mathrm{Zn}$ L bond lengths. Column 3, root mean square deviation for heavy atom (i.e., non-hydrogen) overlay. Column 4, difference between experimental and computed $\mathrm{Zn}-\mathrm{Zn}$ distance (a negative value implies a shorter computed value). All numerical data in $\AA$

\begin{tabular}{|c|c|c|c|}
\hline Refcode & M-L(rmsd) & Heavy atom rmsd & $\mathrm{Zn}-\mathrm{Zn}$ diff \\
\hline ABOWUL & 0.03 & 0.62 & -0.07 \\
\hline ВОНХОМ & 0.02 & 0.30 & -0.16 \\
\hline BOHXUS & 0.01 & 0.45 & -0.13 \\
\hline DAYNEX & 0.01 & 0.95 & -0.21 \\
\hline DOYZIA & 0.02 & 0.45 & -0.06 \\
\hline DUJVOU & 0.01 & 0.57 & -0.17 \\
\hline DUPXUI & 0.04 & 0.47 & -0.13 \\
\hline EBEPAC & 0.03 & 0.87 & -0.14 \\
\hline FACQOQ & 0.02 & 1.37 & -0.08 \\
\hline FOWLAF & 0.04 & 0.55 & -0.17 \\
\hline FOWLOT & 0.02 & 0.75 & -0.08 \\
\hline IJODOB & 0.04 & 0.58 & -0.15 \\
\hline INIBAJ & 0.02 & 0.29 & -0.15 \\
\hline INIZOU & 0.02 & 0.32 & -0.15 \\
\hline IRATAX & 0.02 & 0.49 & -0.16 \\
\hline IRATIF & 0.02 & 0.59 & -0.16 \\
\hline KIKXIM & 0.04 & 0.36 & 0.05 \\
\hline KUSHIQ & 0.03 & 0.90 & -0.16 \\
\hline LIMWUZ & 0.03 & 0.72 & -0.12 \\
\hline NEHZUV & 0.03 & 0.29 & -0.03 \\
\hline NEHZUV01 & 0.02 & 0.13 & -0.06 \\
\hline OGANIU & 0.04 & 0.68 & -0.12 \\
\hline ONASOM & 0.03 & 0.35 & -0.04 \\
\hline ONATAZ & 0.03 & 0.52 & -0.04 \\
\hline QATQAF & 0.03 & 0.92 & -0.09 \\
\hline QETGAY & 0.02 & 0.26 & -0.15 \\
\hline RUDWUJ & 0.02 & 0.75 & -0.14 \\
\hline RUGVOF & 0.02 & 0.92 & -0.13 \\
\hline SADDUY & 0.02 & 0.93 & -0.15 \\
\hline TAHYEI & 0.02 & 0.39 & -0.05 \\
\hline TAYFIJ & 0.04 & 0.83 & -0.03 \\
\hline XAYKOY & 0.05 & 0.35 & -0.04 \\
\hline
\end{tabular}

benzene goes to the pore face opposite the third and the experimental solid-state packing is realized. Sometimes, the fourth benzene goes to the same face as the third and pushes the latter out such that it interacts with the $\mathrm{NMe}_{3}$ groups on the pore periphery. In a periodic system, this benzene would be replicated near the 'vacant' face and, once again, we would deduce that this is the more favorable position and the experimental packing would be recovered once again. Visual inspection of a space-filling representation confirms that there is insufficient space to accommodate a fifth benzene. Finally, a number of short $300 \mathrm{~K} \mathrm{MD}$ annealing simulations were run which confirm that the four benzenes are located in reasonably stable positions.

A similar energy minimization procedure was followed for $\mathrm{dmf}$ and, to a large extent, the $\mathrm{dmf}$ molecules spontaneously migrated to their experimentally-observed positions. However, while in general, the dmf methyl groups are oriented toward the bdc phenyl rings with the carbonyl groups oriented toward dabco pillars, other orientations of dmf molecules have very similar energies and the barrier to rotation is low.

To test the modeling further, a fifth dmf molecule was added. Energy minimization leads to the fifth dmf being captured in a local minimum within the pore. However, a short MD simulated-annealing run shows that even the 1-pore model cannot sustain five $\mathrm{dmf}$ molecules and one is spontaneously pushed out through the relatively unrestricted face formed by bdc linkers. However, in the process, the 1-pore model becomes very badly distorted.

Consequently, we took the 9-pore model and removed the $\mathrm{dmf}$ molecules from all but the central pore. A fifth dmf was then added, the energy minimized and a short MD annealing run carried out. As for the 1-pore model, one of the dmfs is spontaneously ejected from the pore (Fig. 11).

The motions of the dmf molecules are displayed graphically in Fig. 12 which shows the change in the distances between the amide $\mathrm{N}$ atoms of each $\mathrm{dmf}$ molecule and a nearby $\mathrm{Zn}$ atom. Three $\mathrm{dmf}$ molecules barely move while $\mathrm{N} 1$ is the one which is ejected. N2 adjusts its position to generate the approximately tetrahedral arrangement of $\mathrm{dmf}$ molecules in the pore, consistent with the experimental arrangement.

A shortcoming of the preceding series of calculations is the lack of any energetic information. Thus, while we can derive an idea of the maximum loading of adsorbate that an isolated pore can tolerate, we cannot predict the energetic consequences of this loading in the actual MOF since we do not

\section{$\left[\mathrm{Zn}_{\mathbf{2}}(\mathrm{bdc})_{2}(\mathrm{dabco})\right]$ Pore Model}

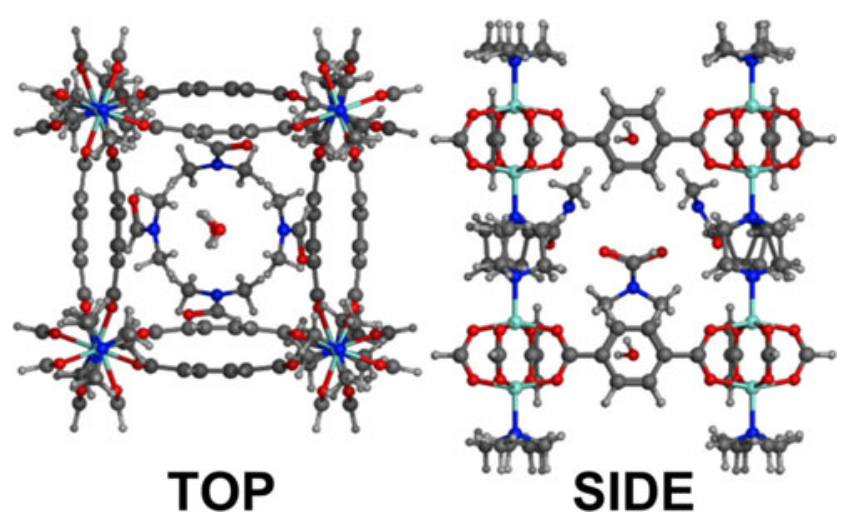

Fig. 9 Starting pore model for MM optimizations derived from X-ray diffraction study. Positions of experimental water molecules are shown but waters are not included in the MM calculations 
Fig. 10 Development of the 'breathing' for

$\left[\mathrm{Zn}(\mathrm{bdc})_{2}(\text { dabco })\right]_{\mathrm{n}}$.solvate. Left column displays the single pore starting model for MM

optimization. Central column shows the ZPW-FF optimized structure of the single pore model. The right column shows the ZPW-FF optimized structure of the central pore of the nine-pore $3 \times 3$ grid
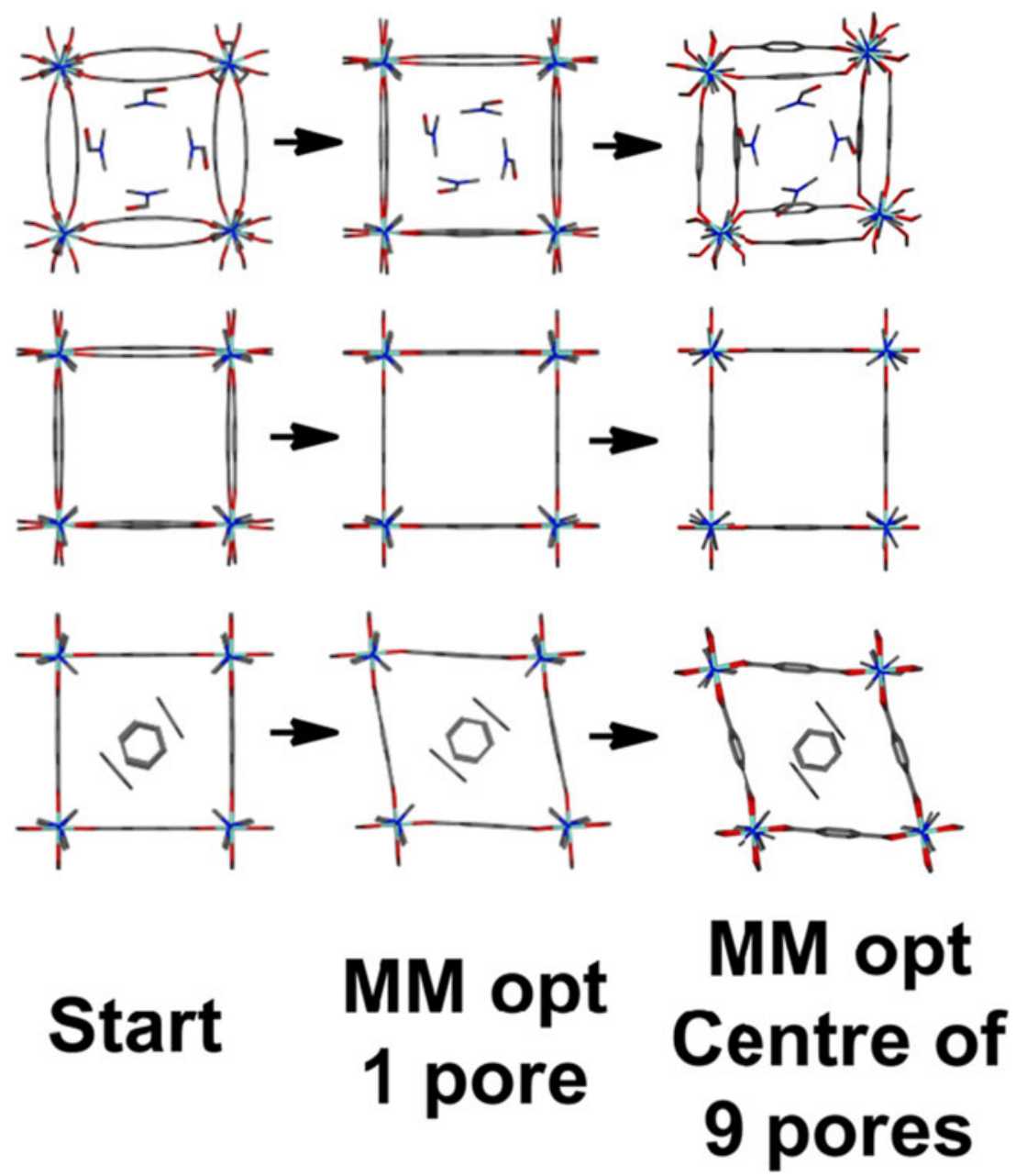

include a full treatment of the pore surroundings. Thus, we cannot deal with the effect of, for example, the external concentration of adsorbate. This requires a more sophisticated dynamics treatment along the lines of that reported by Grosh
Fig. 11 ZPW-FF simulated annealing starting from the energy-minimized, 9-pore model with the central pore containing the four original dmf molecules (in yellow) plus a fifth $\operatorname{dmf}$ (in cyan). After simulated annealing (bottom left), one of the dmf molecules exits the pore (right, highlighted by the dotted red ellipse)

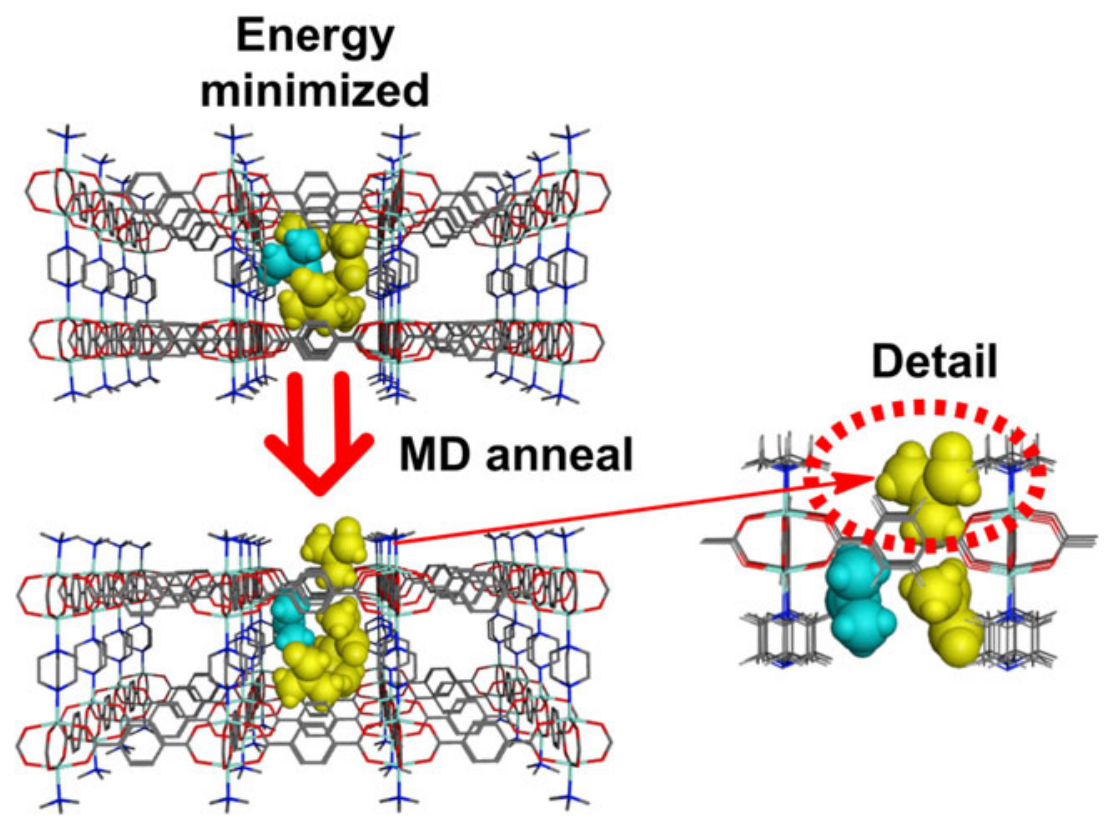




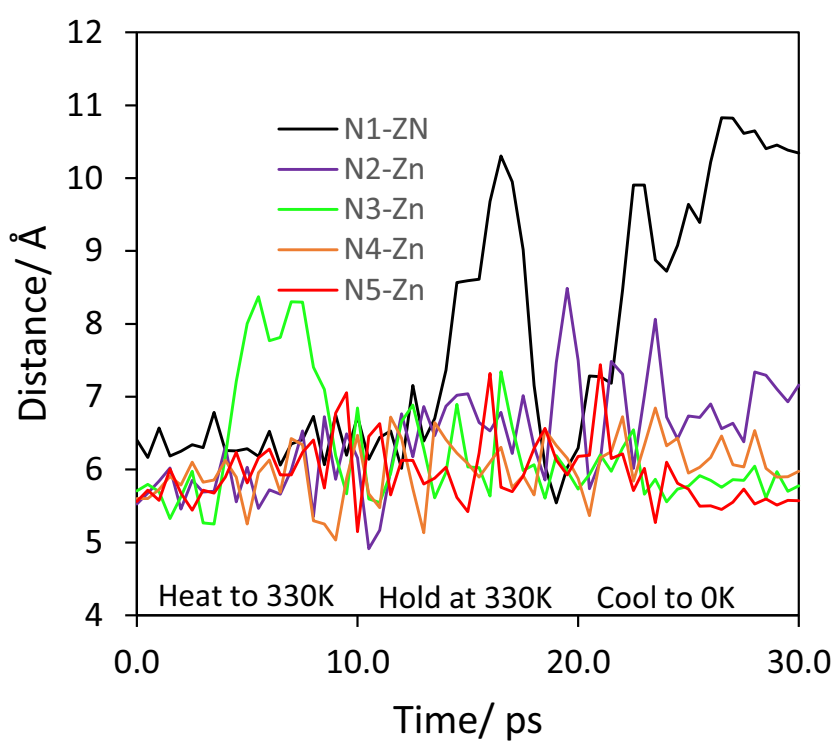

Fig. 12 Plot of distances from a corner zinc center to the $\mathrm{N}$ atom of the five $d m f$ molecules in the central pore of the $3 \times 3$ grid during the simulated annealing MD run showing how one of the dmf molecules (N1) is spontaneously ejected from the central pore

and Paesani [63]. However, the present study at least demonstrates that the underlying ZPW-FF should provide an excellent platform for such studies and we will report the results in due course.

\section{Conclusions}

A new experimentally-refined, first-principles force field has been developed for the zinc paddlewheel motif including pyridyl and amine capping groups. The ZPW-FF parameters are based on the Merck molecular FF (MMFF) extended with additional ligand field molecular mechanics (LFMM) parameters as implemented in DommiMOE, our extended version of the molecular operating environment. Given the absence of explicit d-electron effects for $\mathrm{d}^{10} \mathrm{Zn}$ (II) centers, the parameters could easily be ported to other software codes which support the MMFF and LFMM potential energy functional forms.

The new ZPW-FF is based on a much larger set of training and validation systems than other MOF FFs. It accurately reproduces the DFT-computed structures for small model ZPW systems including uncapped, mono-, and di-capped complexes. This includes the first ever empirical modeling of the high-symmetry transition state structures for the uncapped $\left[\mathrm{Zn}_{2}\left(\mathrm{O}_{2} \mathrm{CR}\right)_{4}\right]$ systems. With a minor refinement of Morse function reference distances for $\mathrm{Zn}-\mathrm{O}$ and $\mathrm{Zn}-\mathrm{N}$ bonds, excellent agreement with the structures of 32 crystallographically-characterized ZPW systems is also obtained.

The ZPW-FF is designed to accurately model the local structure of zinc centers coordinated to bridging carboxylate and monodentate $\mathrm{N}$ donor ligands and can be applied equally well to isolated 'molecular' ZPW systems or to materials such as MOFs with multiple ZPW units. This permits us to construct aperiodic models of MOF pores and explore issues such as whether the behavior observed in the bulk is a function of an individual pore or a set of pores.

The new force field was therefore applied to pore models of the archetypal flexible MOF $\left[\mathrm{Zn}_{2}(\mathrm{bdc})_{2}(\mathrm{dabco})\right]_{\mathrm{n}}$. The dimensions of the calculated structure of an empty single pore are identical to the experimental unit cell parameters of the extended solid. The framework structure is also sensitive to adsorbed solvent and a 1-pore model already gives a qualitatively correct picture of the effect of including four $\mathrm{dmf}$ or four benzene molecules. The latter calculations start from the Xray crystallographic coordinates but the number and orientation of solvent molecules is also predicted by systematically adding solvent molecules to the pore, energy minimizing and then, if necessary, carrying out short annealing MD runs. The four benzene molecules essentially occupy their experimental positions spontaneously. The dmf molecules show some variability in orientation but the energetic consequences are minor. MD simulations further show that any attempt to add a fifth dmf molecule to the pore will result in one being spontaneously ejected.

Overall, the ZPW-FF performs extremely well, at least for $\left[\mathrm{Zn}(\mathrm{bdc})_{2}(\mathrm{dabco})\right]_{\mathrm{n}}$, and is obviously many orders of magnitude more efficient than DFT. Our next goals are to extend the ZPW-FF to other more complicated MOFs and to develop our own version of a copper paddlewheel force field (CPW-FF) where the important d-electron effects arising from the strongly Jahn-Teller active $\mathrm{d}^{9}$ copper(II) centers will be captured explicitly by the angular overlap model parameters of LFMM $[58,64]$ rather than via conventional FF parameters [20]. Also, while pore models may provide some useful insights, the lack of periodicity means that many important mechanical, thermal and dielectric properties cannot be computed [24]. The LFMM methodology has recently been ported to Tinker [65]. The latter avoids the annual licence fees associated with MOE and will hopefully lead to wider uptake by the academic community although there is much development work to be completed before the Tinker-LF implementation can match the functionality currently available in DommiMOE. On the other hand, the ability to model periodic systems in MOE is limited so it is worth noting that LFMM is also available in DL_POLY, although our application involved Pt binding to DNA [62]. We will report on the performance of ZPW-FF for periodic systems in due course.

Acknowledgments The authors gratefully acknowledge the generous support of Chemical Computing Group. KAHA acknowledges the support of the King Abdulaziz University and the Ministry of Higher Education, Kingdom of Saudi Arabia for the provision of a $\mathrm{PhD}$ scholarship. 
Open Access This article is distributed under the terms of the Creative Commons Attribution 4.0 International License (http:// creativecommons.org/licenses/by/4.0/), which permits unrestricted use, distribution, and reproduction in any medium, provided you give appropriate credit to the original author(s) and the source, provide a link to the Creative Commons license, and indicate if changes were made.

\section{References}

1. MacGillivray LR (2010) Metal-organic frameworks: design and application. Wiley, Hoboken

2. Czaja AU, Trukhan N, Mueller U (2009) Industrial applications of metal-organic frameworks. Chem Soc Rev 38(5):1284-1293. doi: $10.1039 / \mathrm{b} 804680 \mathrm{~h}$

3. Horike S, Shimomura S, Kitagawa S (2009) Soft porous crystals. Nat Chem 1(9):695-704

4. Schneemann A, Bon V, Schwedler I, Senkovska I, Kaskel S, Fischer RA (2014) Flexible metal-organic frameworks. Chem Soc Rev 43(16):6062-6096. doi:10.1039/c4cs00101j

5. Sarkisov L, Martin RL, Haranczyk M, Smit B (2014) On the flexibility of metal-organic frameworks. J Am Chem Soc 136(6):22282231. doi:10.1021/ja411673b

6. Zhai Q-G, Bai N, Li Sn BX, Feng P (2015) Design of pore size and functionality in pillar-layered $\mathrm{Zn}$-triazolate-dicarboxylate frameworks and their high $\mathrm{CO} 2 / \mathrm{CH} 4$ and $\mathrm{C} 2$ hydrocarbons/CH4 selectivity. Inorg Chem 54(20):9862-9868. doi:10.1021/acs.inorgchem. 5b01611

7. Mukherjee S, Joarder B, Desai AV, Manna B, Krishna R, Ghosh SK (2015) Exploiting framework flexibility of a metal-organic framework for selective adsorption of styrene over ethylbenzene. Inorg Chem 54(9):4403-4408. doi:10.1021/acs.inorgchem.5b00206

8. Li B, Wen H-M, Zhou W, Chen B (2014) Porous metal-organic frameworks for gas storage and separation: what, how, and why? J Phys Chem Lett 5(20):3468-3479. doi:10.1021/jz501586e

9. Hawes CS, Nolvachai Y, Kulsing C, Knowles GP, Chaffee AL, Marriott PJ, Batten SR, Turner DR (2014) Metal-organic frameworks as stationary phases for mixed-mode separation applications. Chem Commun 50(28):3735-3737. doi:10.1039/C4CC00933A

10. Liu D, Lu K, Poon C, Lin W (2013) Metal-organic frameworks as sensory materials and imaging agents. Inorg Chem 53(4):19161924. doi:10.1021/ic402194c

11. Yue Y, Rabone JA, Liu H, Mahurin SM, Li M-R, Wang H, Lu Z, Chen B, Wang J, Fang Y, Dai S (2015) A flexible metal-organic framework: guest molecules controlled dynamic gas adsorption. J Phys Chem C 119(17):9442-9449. doi: 10.1021/acs.jpcc.5b02359

12. Dybtsev DN, Chun H, Kim K (2004) Rigid and flexible: a highly porous metal-organic framework with unusual guest-dependent dynamic behavior. Angew Chem Int Ed 43(38):5033-5036. doi:10. 1002/anie.200460712

13. Odoh SO, Cramer CJ, Truhlar DG, Gagliardi L (2015) Quantumchemical characterization of the properties and reactivities of metal-organic frameworks. Chem Rev 115(12):6051-6111. doi:10. 1021/cr500551h

14. Chen L, Mowat JPS, Fairen-Jimenez D, Morrison CA, Thompson SP, Wright PA, Düren T (2013) Elucidating the breathing of the metal-organic framework MIL-53(Sc) with ab initio molecular dynamics simulations and in situ X-ray powder diffraction experiments. J Am Chem Soc 135(42):15763-15773. doi:10.1021/ ja403453g

15. Colon YJ, Snurr RQ (2014) High-throughput computational screening of metal-organic frameworks. Chem Soc Rev 43(16): 5735-5749. doi:10.1039/c4cs00070f
16. Gee JA, Sholl DS (2015) Prediction of adsorption properties of cyclic hydrocarbons in MOFs using DFT-derived force fields. J Phys Chem C 119(29):16920-16926. doi:10.1021/acs.jpcc. 5 b03147

17. Wu H, Simmons JM, Liu Y, Brown CM, Wang X-S, Ma S, Peterson VK, Southon PD, Kepert CJ, Zhou H-C, Yildirim T, Zhou W (2010) Metal-organic frameworks with exceptionally high methane uptake: where and how is methane stored? Chem Eur J 16(17): 5205-5214. doi:10.1002/chem.200902719

18. Rappe AK, Casewit CJ, Colwell KS, Goddard WA, Skiff WM (1992) Uff, a full periodic-table force-field for molecular mechanics and molecular-dynamics simulations. J Am Chem Soc 114(25): 10024-10035

19. Mayo SL, Olafson BD, Goddard WA (1990) Dreiding — a generic force-field for molecular simulations. J Phys Chem 94(26):88978909

20. Tafipolsky M, Amirjalayer S, Schmid R (2010) First-principlesderived force field for copper paddle-wheel-based metal-organic frameworks. J Phys Chem C 114(34):14402-14409. doi:10.1021/ jp104441d

21. Haldoupis E, Borycz J, Shi H, Vogiatzis KD, Bai P, Queen WL, Gagliardi L, Siepmann JI (2015) Ab initio derived force fields for predicting $\mathrm{CO} 2$ adsorption and accessibility of metal sites in the metal-organic frameworks M-MOF-74 $(\mathrm{M}=\mathrm{Mn}, \mathrm{Co}, \mathrm{Ni}, \mathrm{Cu}) . \mathrm{J}$ Phys Chem C 119(28):16058-16071. doi:10.1021/acs.jpcc. 5 b03700

22. Bureekaew S, Amirjalayer S, Tafipolsky M, Spickermann C, Roy TK, Schmid R (2013) MOF-FF - a flexible first-principles derived force field for metal-organic frameworks. Physica Status Solidi BBasic Solid State Phys 250(6):1128-1141. doi:10.1002/pssb. 201248460

23. Tafipolsky M, Schmid R (2009) Systematic first principles parameterization of force fields for metal-organic frameworks using a genetic algorithm approach. J Phys Chem B 113(5):1341-1352. doi:10.1021/jp807487f

24. Bristow JK, Tiana D, Walsh A (2014) Transferable force field for metal-organic frameworks from first-principles: BTW-FF. J Chem Theory Comput 10(10):4644-4652. doi: $10.1021 / \mathrm{ct} 500515 \mathrm{~h}$

25. Addicoat MA, Vankova N, Akter IF, Heine T (2014) Extension of the universal force field to metal-organic frameworks. J Chem Theory Comput 10(2):880-891. doi:10.1021/ct400952t

26. Garcia-Perez E, Serra-Crespo P, Hamad S, Kapteijn F, Gascon J (2014) Molecular simulation of gas adsorption and diffusion in a breathing MOF using a rigid force field. PCCP 16(30):16060 16066. doi: $10.1039 / \mathrm{c} 3 \mathrm{cp} 55416 \mathrm{c}$

27. Ćendić M, Matović ZD, Deeth RJ (2013) Molecular modeling for $\mathrm{Cu}(\mathrm{II})$-aminopolycarboxylate complexes: structures, conformational energies, and ligand binding affinities. J Comput Chem 34(31): 2687-2696. doi:10.1002/jcc. 23437

28. Handley CM, Deeth RJ (2012) A multi-objective approach to force field optimization: structures and spin state energetics of $\mathrm{d}^{6} \mathrm{Fe}(\mathrm{II})$ complexes. J Chem Theory Comput 8(1):194-202. doi:10.1021/ ct200584a

29. Brodbeck R, Deeth RJ (2011) Extending ligand field molecular mechanics to modelling organometallic p-bonded systems: applications to ruthenium-arenes. Dalton Trans 40(42):11147-11155. doi: 10.1039/c1dt10794a

30. Deeth RJ, Anastasi AE, Wilcockson MJ (2010) An in silico design tool for Fe(II) spin crossover and light-induced excited spin statetrapped complexes. J Am Chem Soc 132(20):6876-6877. doi:10. 1021/ja1007323

31. Deeth RJ, Anastasi A, Diedrich C, Randell K (2009) Molecular modelling for transition metal complexes: dealing with d-electron effects. Coord Chem Rev 253:795-816 
32. Anastasi A, Deeth RJ (2009) Capturing the trans influence in lowspin $\mathrm{d}^{8}$ square-planar platinum(II) systems using molecular mechanics. J Chem Theory Comput 5:2339-2352

33. Diedrich C, Deeth RJ (2008) On the performance of ligand field molecular mechanics for model complexes containing the peroxido-bridged $\left[\mathrm{Cu}_{2} \mathrm{O}_{2}\right]^{2+}$ center. Inorg Chem 47:2494-2506

34. Bentz A, Comba P, Deeth R, Al E (2008) Modeling of the various minima on the potential energy surface of bispidine copper(II) complexes: a further test for ligand field molecular mechanics. Inorg Chem 47(20):9518-9527. doi:10.1021/ic8011052

35. Deeth RJ (2007) A comprehensive molecular mechanics model for oxidised type I copper proteins: active site structures, strain energies and entatic bulging. Inorg Chem 46:4492-4503

36. Neese F, Becker U, Ganiouchine D, Koßmann S, Petrenko T, Riplinger C, Wennmohs F (2014) ORCA: program version 3.0. ORCA 3.0.1 edn. Max Planck Institute for Chemnical Energy Conversion, Muellheim

37. Becke AD (1988) Density-functional exchange-energy approximation with correct asymptotic behavior. Phys Rev A 38(6):30983100

38. Perdew JP, Yue W (1986) Accurate and simple density functional for the electronic exchange energy - generalized gradient approximation. Phys Rev B-Condensed Matter 33(12):8800-8802

39. Schaefer A, Horn H, Ahlrichs R (1992) Ahlrichs def2 basis sets. J Chem Phys 97:2571

40. Hocking RK, Deeth RJ, Hambley TW (2007) DFT study of the systematic variations in metal-ligand bond lengths of coordination complexes: the crucial role of the condensed phase. Inorg Chem 46(20):8238-8244

41. Klamt A, Jones V (1996) COSMO: ref 3. J Chem Phys 105:9972

42. Klamt A (1995) COSMO: ref 2. J Phys Chem 99:2224

43. Klamt A, Schüürmann G (1993) COSMO: ref 1. J Chem Soc Perkin Trans 2:799

44. Deeth RJ, Fey N, Williams-Hubbard BJ (2005) DommiMOE: an implementation of ligand field molecular mechanics in the molecular operating environment. J Comput Chem 26:123-130

45. Chemical Computing Group (2011) Molecular operating environment (MOE) 2011.10 edn. Chemical Computing Group, Montreal

46. Deeth RJ, Foulis DL (2002) Analytical derivatives, pi bonding and $\mathrm{d}$-s mixing in the ligand field molecular mechanics method. PCCP 4(18):4292-4297

47. Users are responsible for obtaining a valid MOE licence from Chemical Computing Group

48. Comba P, Remenyi R (2003) Inorganic and bioinorganic molecular mechanics modeling - the problem of the force field parameterization. Coord Chem Rev 238:9-20

49. Norrby PO, Brandt P (2001) Deriving force field parameters for coordination complexes. Coord Chem Rev 212:79-109

50. Deeth RJ, Hearnshaw LJA (2006) Molecular modelling of JahnTeller distortions in $\mathrm{Cu}(\mathrm{II}) \mathrm{N}_{6}$ complexes: elongations, compressions and the pathways in between. Dalton Trans:1092-1100

51. Zhao X, He H, Hu T, Dai F, Sun D (2009) Interpenetrating polyhedral MOF with a primitive cubic network based on supermolecular building blocks constructed of a semirigid C3-symmetric carboxylate ligand. Inorg Chem 48(17):8057-8059. doi:10.1021/ ic $901004 \mathrm{~m}$

52. Houghton BJ, Deeth RJ (2014) Spin-state energetics of feii complexes - the continuing voyage through the density functional minefield. Eur J Inorg Chem 2014(27):4573-4580. doi:10.1002/ ejic. 201402253
53. Baerends EJ, Ziegler T, Autschbach J, Bashford D, Bérces A, Bickelhaupt FM, Bo C, Boerrigter PM, Cavallo L, Chong DP, Deng L, Dickson RM, Ellis DE, van Faassen M, Fan L, Fischer TH, Fonseca Guerra C, Ghysels A, Giammona A, van Gisbergen SJA, Götz AW, Groeneveld JA, Gritsenko OV, Grüning M, Gusarov S, Harris FE, van den Hoek P, Jacob CR, Jacobsen H, Jensen L, Kaminski JW, van Kessel G, Kootstra F, Kovalenko A, Krykunov MV, van Lenthe E, McCormack DA, Michalak A, Mitoraj M, Neugebauer J, Nicu VP, Noodleman L, Osinga VP, Patchkovskii S, Philipsen PHT, Post D, Pye CC, Ravenek W, Rodríguez JI, Ros P, Schipper PRT, Schreckenbach G, Seldenthuis JS, Seth M, Snijders JG, Solà M, Swart M, Swerhone D, te Velde G, Vernooijs P, Versluis L, Visscher L, Visser O, Wang F, Wesolowski TA, van Wezenbeek EM, Wiesenekker G, Wolff SK, Woo TK, Yakovlev AL (2012) ADF2012.01. SCM, theoretical chemistry. Vrije Universiteit, Amsterdam

54. van Lenthe E, Ehlers AE, Baerends EJ (1999) ADF: ZORA ref 5. J Chem Phys 110:8943

55. Nazarian D, Ganesh P, Sholl DS (2015) Benchmarking density functional theory predictions of framework structures and properties in a chemically diverse test set of metal-organic frameworks. J Mater Chem A 3(44):22432-22440. doi:10.1039/C5TA03864B

56. Bureekaew S, Amirjalayer S, Schmid R (2012) Orbital directing effects in copper and zinc based paddle-wheel metal organic frameworks: the origin of flexibility. J Mater Chem 22(20):10249-10254. doi:10.1039/c2jm15778k

57. Allured VS, Kelly CM, Landis CR (1991) Shapes empirical forcefield - new treatment of angular potentials and its application to square-planar transition- metal complexes. J Am Chem Soc 113(1):1-12

58. Deeth RJ (2001) The ligand field molecular mechanics model and the stereoelectronic effects of $d$ and s electrons. Coord Chem Rev 212:11-34

59. Comba P, Hambley TW (1995) Molecular modeling of inorganic compounds. VCH, Weinheim

60. Deeth RJ (2008) General molecular mechanics method for transition metal carboxylates and its application to the multiple coordination modes in mono- and dinuclear $\mathrm{Mn}$ (II) complexes. Inorg Chem 47(15):6711-6725. doi:10.1021/ic800313s

61. Chemical Computing Group (2013) Molecular operating environment (MOE) 2013.0801 edn. Chemical Computing Group, Montreal

62. Tai H-C, Brodbeck R, Kasparkova J, Farrer NJ, Brabec V, Sadler PJ, Deeth RJ (2012) A combined theoretical and computational study of interstrand DNA guanine-guanine cross-linking by trans[Pt(pyridine)2] derived from the photoactivated prodrug trans, trans, trans-[Pt(N3)2(OH)2(pyridine)2]. Inorg Chem 51:68306841

63. Grosch JS, Paesani F (2012) Molecular-level characterization of the breathing behavior of the jungle-gym-type DMOF-1 metal-organic framework. J Am Chem Soc 134(9):4207-4215. doi:10.1021/ ja2100615

64. Burton VJ, Deeth RJ, Kemp CM, Gilbert PJ (1995) Molecular mechanics for coordination-complexes-the impact of adding d-electron stabilization energies. J Am Chem Soc 117(32):8407-8415

65. Foscato M, Deeth RJ, Jensen VR (2015) Integration of ligand field molecular mechanics in tinker. J Chem Inf Mod 55(6):1282-1290. doi:10.1021/acs.jcim.5b00098 